\title{
Time Evolution of Decay Spectrum in $K^{0}, \overline{K^{0}} \rightarrow \pi^{+} \pi^{-} e^{+} e^{-}$
}

\author{
L. M. Sehgal and J. van Leusen \\ Institute of Theoretical Physics, RWTH Aachen, D-52056 Aachen, Germany
}

\begin{abstract}
We consider the decay $K^{0}\left(\overline{K^{0}}\right) \rightarrow \pi^{+} \pi^{-} e^{+} e^{-}$of a neutral $K$ meson prepared in a state of strangeness $+1(-1)$. The time evolution of the state produces remarkable time-dependent effects in the angular distribution of the $\pi^{+} \pi^{-} e^{+} e^{-}$system. These effects are correlated with the time-dependence of the photon polarization in the radiative decay $K^{0}\left(\overline{K^{0}}\right) \rightarrow \pi^{+} \pi^{-} \gamma$. We study, in particular, the $C P$-odd, $T$-odd term in the distribution $d \Gamma / d \phi$ of the angle between the $\pi^{+} \pi^{-}$and the $e^{+} e^{-}$planes. We also give the spectrum in the case that the decaying meson is an incoherent mixture of $K^{0}$ and $\overline{K^{0}}$, and discuss the case of $K_{S}$ regeneration in a $K_{L}$ beam.
\end{abstract}

\section{Introduction}

The KTeV experiment [1] has measured a large $C P$-violating, $T$-odd asymmetry in the decay $K_{L} \rightarrow \pi^{+} \pi^{-} e^{+} e^{-}$, in quantitative agreement with a prediction made some years ago $[2,3]$. The origin of this effect lies in the amplitude of the radiative decay $K_{L} \rightarrow \pi^{+} \pi^{-} \gamma$, which contains a bremsstrahlung term proportional to $\eta_{+-}$, as well as a $C P$-conserving direct emission term of magnetic dipole character [4]. The interference of the odd electric multipoles $E 1, E 3, E 5 \cdots$ present in the bremsstrahlung amplitude, which all have $C P=+1$, with the magnetic $M 1$ multipole of $C P=-1$, produces $C P$-violating components in the polarization state (Stokes vector) of the photon [5]. The Dalitz pair process $K_{L} \rightarrow \pi^{+} \pi^{-} e^{+} e^{-}$acts as an analyser of the photon polarization, exposing the $C P$-odd, $T$-odd component of the Stokes vector. The specific distribution that reveals the $C P$-violation is

$$
\frac{d \Gamma}{d \phi} \sim 1-\left(\Sigma_{3} \cos 2 \phi+\Sigma_{1} \sin 2 \phi\right)
$$


where $\phi$ is the angle between the $\pi^{+} \pi^{-}$and $e^{+} e^{-}$planes. The last term in Eq. (1) is $C P$-odd and $T$-odd, and produces an asymmetry

$$
\mathcal{A}_{\phi}=\frac{\left(\int_{0}^{\pi / 2}-\int_{\pi / 2}^{\pi}+\int_{\pi}^{3 \pi / 2}-\int_{3 \pi / 2}^{2 \pi}\right) \frac{d \Gamma}{d \phi} d \phi}{\left(\int_{0}^{\pi / 2}+\int_{\pi / 2}^{\pi}+\int_{\pi}^{3 \pi / 2}+\int_{3 \pi / 2}^{2 \pi}\right) \frac{d \Gamma}{d \phi} d \phi}=-\frac{2}{\pi} \Sigma_{1} .
$$

The measured value [1] $\left|\mathcal{A}_{\phi}\right|=(13.6 \pm 2.5 \pm 1.2) \%$ is in excellent agreement with the prediction $[2,3]$ of $14 \%$.

In a recent report [6], the NA48 collaboration, while confirming the large $C P$-violating effect in $K_{L} \rightarrow \pi^{+} \pi^{-} e^{+} e^{-}$, has also studied the decay $K_{S} \rightarrow$ $\pi^{+} \pi^{-} e^{+} e^{-}$, finding no asymmetry in this case. This is entirely consistent with the fact that the amplitude $K_{S} \rightarrow \pi^{+} \pi^{-} \gamma$ is accurately reproduced by bremsstrahlung alone, so that no electric-magnetic interference is expected.

An interesting question raised by the above observations is the following: How does the asymmetry $\mathcal{A}_{\phi}$ evolve with time if the neutral $K$ meson is prepared in an initial state $K^{0}$ or $\overline{K^{0}}$ of definite strangeness? How does the value of $\mathcal{A}_{\phi}$ evolve from zero at short times (when the state decays like $K_{S}$ ) to the value $-14 \%$ at large times (when the state is essentially $K_{L}$ )? Finally, what type of evolution is expected in the decay of an incoherent $K^{0}-\overline{K^{0}}$ mixture, such as an untagged neutral kaon beam originating in the decay $\phi \rightarrow K^{0} \overline{K^{0}}$ ?

This paper answers these questions by analysing in detail the time dependence of the decays $K^{0}, \overline{K^{0}} \rightarrow \pi^{+} \pi^{-} e^{+} e^{-}$. We study the evolution of the full decay spectrum, especially the terms that are odd under $C P$. A comparison is made between the behaviour of beams that are initially $K^{0}, \overline{K^{0}}$ or an incoherent (untagged) mixture.

As a prelude to the discussion of the channel $K^{0}, \overline{K^{0}} \rightarrow \pi^{+} \pi^{-} e^{+} e^{-}$, we analyse in Section 2 the time-dependence of the photon polarization in $K^{0}, \overline{K^{0}} \rightarrow$ $\pi^{+} \pi^{-} \gamma$. This will reveal the behaviour of the $C P$-odd components of the photon Stokes vector, one of which is reflected in the asymmetry $\mathcal{A}_{\phi}$ in $K^{0}, \overline{K^{0}} \rightarrow$ $\pi^{+} \pi^{-} e^{+} e^{-}$, that we discuss in Section 3 .

\section{Time Evolution of Photon Polarization in $K^{0}, \overline{K^{0}} \rightarrow \pi^{+} \pi^{-} \gamma$}

The measured branching ratios and photon energy spectra in the decays $K_{L} \rightarrow$ $\pi^{+} \pi^{-} \gamma$ and $K_{S} \rightarrow \pi^{+} \pi^{-} \gamma[4]$ can be well-described by the matrix elements [2]

$$
\mathcal{M}\left(K_{S} \rightarrow \pi^{+} \pi^{-} \gamma\right)=e f_{S}\left[\frac{\epsilon \cdot p_{+}}{k \cdot p_{+}}-\frac{\epsilon \cdot p_{-}}{k \cdot p_{-}}\right]
$$




$$
\mathcal{M}\left(K_{L} \rightarrow \pi^{+} \pi^{-} \gamma\right)=e f_{L}\left[\frac{\epsilon \cdot p_{+}}{k \cdot p_{+}}-\frac{\epsilon \cdot p_{-}}{k \cdot p_{-}}\right]+e \frac{f_{D E}}{M_{K}{ }^{4}} \epsilon_{\mu \nu \rho \sigma} \epsilon^{\mu} k^{\nu} p_{+}{ }^{\rho} p_{-}{ }^{\sigma}
$$

where

$$
\begin{gathered}
f_{S}=\left|f_{S}\right| e^{i \delta_{0}\left(s=M_{K}^{2}\right)}, f_{L}=\eta_{+-} f_{S} \\
f_{D E}=\left|f_{S}\right| g_{M 1}, \quad g_{M 1}=i(0.76) e^{i \delta_{1}(s)}
\end{gathered}
$$

Here $\delta_{0,1}$ are the $\pi^{+} \pi^{-}$phase shifts in the $I=0 s$-wave and $I=1 p$-wave channel, respectively. Introducing the notation [5]

$$
\begin{aligned}
\mathcal{M}\left(K_{S, L} \rightarrow \pi^{+} \pi^{-} \gamma\right)= & \frac{e\left|f_{S}\right|}{M_{K}{ }^{4}}\left\{E_{S, L}(\omega, \cos \theta)\left[\epsilon \cdot p_{+} k \cdot p_{-}-\epsilon \cdot p_{-} k \cdot p_{+}\right]\right. \\
& \left.+M_{S, L}(\omega, \cos \theta) \epsilon_{\mu \nu \rho \sigma} \epsilon^{\mu} k^{\nu} p_{+}{ }^{\rho} p_{-}{ }^{\sigma}\right\}
\end{aligned}
$$

we have for the electric and magnetic amplitudes

$$
\begin{gathered}
E_{S}=\left(\frac{2 M_{K}}{\omega}\right)^{2} \frac{e^{i \delta_{0}\left(s=M_{K}^{2}\right)}}{1-\beta^{2} \cos ^{2} \theta}, M_{S}=0 \\
E_{L}=\left(\frac{2 M_{K}}{\omega}\right)^{2} \frac{\eta_{+-} e^{i \delta_{0}\left(s=M_{K}^{2}\right)}}{1-\beta^{2} \cos ^{2} \theta}, M_{L}=i(0.76) e^{i \delta_{1}(s)} .
\end{gathered}
$$

As in [5], $\omega$ is the photon energy in the kaon rest frame, and $\theta$ is the angle of the $\pi^{+}$relative to the photon in the $\pi^{+} \pi^{-}$c.m. frame. Noting that the strangeness eigenstates $K^{0}$ and $\overline{K^{0}}$ can be written as

$$
\begin{aligned}
& K^{0}=\left(K_{S}+K_{L}\right) / \mathcal{N} \\
& \overline{K^{0}}=\left(K_{S}-K_{L}\right) / \overline{\mathcal{N}},
\end{aligned}
$$

the decay amplitudes for $K^{0}$ and $\overline{K^{0}}$ at time $t$ are

$$
\begin{aligned}
\mathcal{M}\left(K^{0}(t) \rightarrow \pi^{+} \pi^{-} \gamma\right) \sim & \left\{E(t, \omega, \cos \theta)\left[\epsilon \cdot p_{+} k \cdot p_{-}-\epsilon \cdot p_{-} k \cdot p_{+}\right]\right. \\
& \left.+M(t, \omega, \cos \theta) \epsilon_{\mu \nu \rho \sigma} \epsilon^{\mu} k^{\nu} p_{+}{ }^{\rho} p_{-}{ }^{\sigma}\right\} \\
\mathcal{M}\left(\overline{K^{0}}(t) \rightarrow \pi^{+} \pi^{-} \gamma\right) \sim & \left\{\bar{E}(t, \omega, \cos \theta)\left[\epsilon \cdot p_{+} k \cdot p_{-}-\epsilon \cdot p_{-} k \cdot p_{+}\right]\right. \\
& \left.+\bar{M}(t, \omega, \cos \theta) \epsilon_{\mu \nu \rho \sigma} \epsilon^{\mu} k^{\nu} p_{+}{ }^{\rho} p_{-}{ }^{\sigma}\right\}
\end{aligned}
$$

where

$$
\begin{aligned}
E & =e^{-i \lambda_{S} t} E_{S}(\omega, \cos \theta)+e^{-i \lambda_{L} t} E_{L}(\omega, \cos \theta) \\
M & =e^{-i \lambda_{L} t} M_{L}(\omega, \cos \theta)
\end{aligned}
$$




$$
\begin{aligned}
\bar{E} & =e^{-i \lambda_{S} t} E_{S}(\omega, \cos \theta)-e^{-i \lambda_{L} t} E_{L}(\omega, \cos \theta) \\
\bar{M} & =-e^{-i \lambda_{L} t} M_{L}(\omega, \cos \theta)
\end{aligned}
$$

with $\lambda_{S, L}=m_{S, L}-\frac{i}{2} \Gamma_{S, L}, t$ being the proper time.

The amplitudes (8) determine the Stokes vector of the photon in $K^{0}, \overline{K^{0}} \rightarrow$ $\pi^{+} \pi^{-} \gamma$. For $K^{0}$ decay, the components of the Stokes vector $\vec{S}=\left(S_{1}, S_{2}, S_{3}\right)$ at a time $t$ are

$$
\begin{aligned}
& S_{1}(t)=\frac{2 \operatorname{Re}\left[E^{*}(t) M(t)\right]}{|E(t)|^{2}+|M(t)|^{2}} \\
& S_{2}(t)=\frac{2 \operatorname{Im}\left[E^{*}(t) M(t)\right]}{|E(t)|^{2}+|M(t)|^{2}} \\
& S_{3}(t)=\frac{|E(t)|^{2}-|M(t)|^{2}}{|E(t)|^{2}+|M(t)|^{2}} .
\end{aligned}
$$

The corresponding components for $\overline{K^{0}}$ decay are obtained by replacing $E(t) \rightarrow$ $\bar{E}(t), M(t) \rightarrow \bar{M}(t)$.

The physical significance of $S_{2}(t)$ is that it represents the net circular polarization of the photon in $K^{0} \rightarrow \pi^{+} \pi^{-} \gamma$ at time $t$ :

$$
S_{2}(t)=\frac{d \Gamma\left(\lambda_{\gamma}=-1, t\right)-d \Gamma\left(\lambda_{\gamma}=+1, t\right)}{d \Gamma\left(\lambda_{\gamma}=-1, t\right)+d \Gamma\left(\lambda_{\gamma}=+1, t\right)}
$$

The parameters $S_{1}(t)$ and $S_{3}(t)$, on the other hand describe the dependence of the decay rate on the orientation of the polarization vector $\vec{\epsilon}$ relative to $\vec{n}_{\pi}$, the unit vector normal to the $\pi^{+} \pi^{-}$plane:

$$
\frac{d \Gamma(t)}{d \phi} \sim 1-\left(S_{3}(t) \cos 2 \phi+S_{1}(t) \sin 2 \phi\right)
$$

where the coordinates are chosen such that

$$
\vec{k}=(0,0, k), \vec{\epsilon}=(\cos \phi, \sin \phi, 0), \vec{n}_{\pi}=\frac{\vec{p}_{+} \times \vec{p}_{-}}{\left|\vec{p}_{+} \times \vec{p}_{-}\right|}=(1,0,0)
$$

In Figs. 1a and 2a, we show the components $S_{1}(t)$ and $S_{2}(t)$ as functions of photon energy for the decay of an initial $K^{0}$. The corresponding Stokes vector components for an initial $\overline{K^{0}}$ are shown in Figs. $1 \mathrm{~b}$ and $2 \mathrm{~b}$. Notice the intricate interference effect in the time-dependence, particularly in the region $t \sim 10 \tau_{S}$. Of special interest is the limiting case $t \rightarrow \infty$, when the beam is essentially pure $K_{L}$. The Stokes vector components $S_{1}$ and $S_{2}$ then reduce to those shown 
in [5] for the case $K_{L} \rightarrow \pi^{+} \pi^{-} \gamma$. For comparison with the $K^{0}$ and $\overline{K^{0}}$ cases, we have also considered the case of an untagged initial beam, consisting of an incoherent equal mixture of $K^{0}$ and $\overline{K^{0}}$. The Stokes vector is then

$$
\begin{aligned}
\left\langle S_{1}(t)\right\rangle & =\frac{2 \operatorname{Re}\left[E^{*}(t) M(t)+\bar{E}^{*}(t) \bar{M}(t)\right]}{|E(t)|^{2}+|M(t)|^{2}+|\bar{E}(t)|^{2}+|\bar{M}(t)|^{2}} \\
\left\langle S_{2}(t)\right\rangle & =\frac{2 \operatorname{Im}\left[E^{*}(t) M(t)+\bar{E}^{*}(t) \bar{M}(t)\right]}{|E(t)|^{2}+|M(t)|^{2}+|\bar{E}(t)|^{2}+|\bar{M}(t)|^{2}} \\
\left\langle S_{3}(t)\right\rangle & =\frac{|E(t)|^{2}-|M(t)|^{2}+|\bar{E}(t)|^{2}-|\bar{M}(t)|^{2}}{|E(t)|^{2}+|M(t)|^{2}+|\bar{E}(t)|^{2}+|\bar{M}(t)|^{2}} .
\end{aligned}
$$

The time-dependent parameters $\left\langle S_{1}(t)\right\rangle$ and $\left\langle S_{2}(t)\right\rangle$ are plotted in Figs. 1c and 2c. Notice that the strong fluctuations in $S_{1}(t)$ and $S_{2}(t)$ for the $K^{0}$ and $\overline{K^{0}}$ cases are now smoothed out. For $t \gg \tau_{S}$, of course, all three cases give the same Stokes vector, namely that corresponding to $K_{L} \rightarrow \pi^{+} \pi^{-} \gamma$.

The polarization components $\left\langle S_{1}(t)\right\rangle$ and $\left\langle S_{2}(t)\right\rangle$ in the incoherent case have a special significance: they represent $C P$-violating observables at any time $t$. In the model under discussion, they vanish when $\eta_{+-}=0$, which is not the case for the components $S_{1}(t)$ and $S_{2}(t)$ originating from $K^{0}$ or $\overline{K^{0}}$. Furthermore, in the "hermitian limit" [5] (i.e. $\delta_{0}=\delta_{1}=0, \arg \eta_{+-}=\frac{\pi}{2}$ ) the parameter $\left\langle S_{1}(t)\right\rangle$ survives, but $\left\langle S_{2}(t)\right\rangle$ vanishes. This is the hallmark that characterises the observable $\left\langle S_{1}(t)\right\rangle$ as being $C P$-odd, $T$-odd, and the observable $\left\langle S_{2}(t)\right\rangle$ as being $C P$-odd, $T$-even [7].

Of particular relevance in our discussion of $K^{0}, \overline{K^{0}} \rightarrow \pi^{+} \pi^{-} e^{+} e^{-}$is the behaviour of $S_{1}(t, \omega)$ portrayed in Fig. 1 as a function of photon energy $\omega$. This will be found to have a resemblance with the asymmetry $\mathcal{A}_{\phi}\left(t, s_{\pi}\right)$ as a function of the $\pi^{+} \pi^{-}$invariant mass (recall that for $K \rightarrow \pi^{+} \pi^{-} \gamma, s_{\pi}=M_{K}^{2}-2 M_{K} \omega$ ). We now proceed to a systematic analysis of time dependence in the Dalitz pair reaction.

\section{Time Evolution of $K^{0}, \overline{K^{0}} \rightarrow \pi^{+} \pi^{-} e^{+} e^{-}$}

We begin by recalling the matrix element of the long-lived kaon decay $K_{L} \rightarrow$ $\pi^{+} \pi^{-} e^{+} e^{-}$, treating the $e^{+} e^{-}$system as an internal conversion pair associated with the radiative decay $K_{L} \rightarrow \pi^{+} \pi^{-} \gamma[2,3]$. Writing the matrix element in the form

$$
\mathcal{M}\left(K_{L} \rightarrow \pi^{+} \pi^{-} e^{+} e^{-}\right)=-2 \frac{G_{F}}{\sqrt{2}} \sin \theta_{C}\left\{\frac{1}{M_{K}}\left[f\left(p_{+}+p_{-}\right)_{\lambda}+g\left(p_{+}-p_{-}\right)_{\lambda}\right]\right.
$$




$$
\begin{aligned}
& +i \frac{h}{M_{K}^{2}} \epsilon_{\lambda \mu \nu \sigma} p_{K}^{\mu}\left(p_{+}+p_{-}\right)^{\nu}\left(p_{+}-p_{-}\right)^{\sigma} \\
& \times \bar{u}\left(k_{-}\right) \gamma^{\lambda} v\left(k_{+}\right)
\end{aligned}
$$

the form factors $f, g$ and $h$ are related to the parameters $f_{S}, \eta_{+-}$and $g_{M 1}$ of the radiative decay as follows [3]:

$$
\begin{aligned}
& f=C M_{K}^{4}\left|\eta_{+-}\right| e^{i\left(\delta\left(M_{K}^{2}\right)+\varphi_{+-}\right)} \frac{1}{s_{l}} \frac{-4 \beta \cos \theta_{\pi}}{s\left(1-\beta^{2} \cos ^{2} \theta_{\pi}\right)} \\
& g=C M_{K}^{4}\left|\eta_{+-}\right| e^{i\left(\delta\left(M_{K}^{2}\right)+\varphi_{+-}\right)} \frac{1}{s_{l}} \frac{4}{s\left(1-\beta^{2} \cos ^{2} \theta_{\pi}\right)} \\
& h=-C M_{K}^{4} \frac{1}{s_{l}}(0.76) e^{i \delta_{1}\left(s_{\pi}\right)}
\end{aligned}
$$

Here $\theta_{\pi}$ is the angle of the $\pi^{+}$in the $\pi^{+} \pi^{-}$rest frame, relative to the sum of the $e^{+}$and $e^{-}$momenta in that frame; $s_{l}$ is the invariant mass of the lepton pair; $s_{\pi}$ is the $\pi^{+} \pi^{-}$invariant mass; and $s$ is defined as $s=\frac{1}{2}\left(M_{K}^{2}-s_{\pi}-s_{l}\right)$. The constant $C$ is given by

$$
C M_{K}^{4}=\left(-\frac{G_{F}}{\sqrt{2}} \sin \theta_{C} \frac{1}{M_{K}}\right)^{-1} \pi \alpha\left|f_{S}\right|
$$

It is now easy to obtain from the amplitude $\mathcal{M}\left(K_{L} \rightarrow \pi^{+} \pi^{-} e^{+} e^{-}\right)$the corresponding time-dependent amplitudes for $K^{0}$ and $\overline{K^{0}}$ decay by making use of the following artifice: To obtain $\mathcal{M}\left(K^{0}(t) \rightarrow \pi^{+} \pi^{-} e^{+} e^{-}\right)$, we make the replacement

$$
\begin{aligned}
& f \rightarrow f\left(e^{-i \lambda_{L} t}+\frac{1}{\eta_{+-}} e^{-i \lambda_{S} t}\right) / \mathcal{N} \\
& g \rightarrow g\left(e^{-i \lambda_{L} t}+\frac{1}{\eta_{+-}} e^{-i \lambda_{S} t}\right) / \mathcal{N} \\
& h \rightarrow h e^{-i \lambda_{L} t} / \mathcal{N}
\end{aligned}
$$

in Eq. ( 14$)$. Similarly, to obtain $\mathcal{M}\left(\overline{K^{0}}(t) \rightarrow \pi^{+} \pi^{-} e^{+} e^{-}\right)$, we make the replacement

$$
\begin{aligned}
& f \rightarrow f\left(-e^{-i \lambda_{L} t}+\frac{1}{\eta_{+-}} e^{-i \lambda_{S} t}\right) / \overline{\mathcal{N}} \\
& g \rightarrow g\left(-e^{-i \lambda_{L} t}+\frac{1}{\eta_{+-}} e^{-i \lambda_{S} t}\right) / \overline{\mathcal{N}} \\
& h \rightarrow-h e^{-i \lambda_{L} t} / \overline{\mathcal{N}} .
\end{aligned}
$$


The normalization factors $\mathcal{N}$ and $\overline{\mathcal{N}}$ in Eqs. (17) and (18) are those in the states $K^{0}$ and $\overline{K^{0}}$ (Eq. (6)), where $\mathcal{N}=2 p, \overline{\mathcal{N}}=2 q$, with $|p|^{2}-|q|^{2}=\frac{2 \operatorname{Re} \eta_{+-}}{1+\left|\eta_{+-}\right|^{2}}$ and $|p|^{2}+|q|^{2}=1$. With this prescription in mind, we will continue to use the formalism developed in [3] for $K_{L} \rightarrow \pi^{+} \pi^{-} e^{+} e^{-}$, with the understanding that in dealing with $K^{0}$ and $\overline{K^{0}}$ decay, the form factors $f, g$ and $h$ are to be replaced by the time-dependent combinations given in Eqs. (17) and (18).

Following the procedure of Ref. [3], we have calculated the angular distribution of the decays $K^{0}(t) \rightarrow \pi^{+} \pi^{-} e^{+} e^{-}$and $\overline{K^{0}}(t) \rightarrow \pi^{+} \pi^{-} e^{+} e^{-}$in the form

$$
\frac{d \Gamma}{d \cos \theta_{l} d \phi}=K_{1}+K_{2} \cos 2 \theta_{l}+K_{3} \sin ^{2} \theta_{l} \cos 2 \phi+K_{9} \sin ^{2} \theta_{l} \sin 2 \phi
$$

Here $\theta_{l}$ is the angle of $e^{+}$relative to the dipion momentum vector in the $e^{+} e^{-}$ frame; and $\phi$ is the angle between the $\pi^{+} \pi^{-}$and $e^{+} e^{-}$planes. The last term in Eq. (19) is odd under the CP-transformation $\vec{p}_{ \pm} \rightarrow-\vec{p}_{\mp}, \vec{k}_{ \pm} \rightarrow-\vec{k}_{\mp}$, as well as under the $T$-transformation $\vec{p}_{ \pm} \rightarrow-\vec{p}_{ \pm}, \vec{k}_{ \pm} \rightarrow-\vec{k}_{ \pm}$. The timedependent coefficients $K_{2} / K_{1}, K_{3} / K_{1}$ and $K_{9} / K_{1}$ are shown in Fig. 3, where we compare the cases $K^{0}$ and $\overline{K^{0}}$, and also show the result for an incoherent $K^{0}-\overline{K^{0}}$ mixture. This figure depicts the manner in which the coefficients of the angular distribution evolve to their asymptotic values appropriate to the decay $K_{L} \rightarrow \pi^{+} \pi^{-} e^{+} e^{-}$, namely $K_{2} / K_{1}=0.297, K_{3} / K_{1}=0.180, K_{9} / K_{1}=$ $-0.309[3]$.

Integrating Eq. (19) over $\cos \theta_{l}$, we obtain the $\phi$-distribution

$$
\frac{d \Gamma}{d \phi} \sim\left(1-\frac{1}{3} \frac{K_{2}}{K_{1}}\right)+\frac{2}{3}\left(\frac{K_{3}}{K_{1}} \cos 2 \phi+\frac{K_{9}}{K_{1}} \sin 2 \phi\right)
$$

which corresponds to an asymmetry

$$
\mathcal{A}_{\phi}=\frac{2}{\pi} \frac{\frac{2}{3} \frac{K_{9}}{K_{1}}}{1-\frac{1}{3} \frac{K_{2}}{K_{1}}}
$$

The time-dependence of this asymmetry is exhibited in Fig. 4, which is the answer to the question of how this asymmetry evolves from the value zero, appropriate to $K_{S}$ decay, to the value $-14 \%$ observed for $K_{L}$ decay. We have also studied the time-dependent asymmetry $\mathcal{A}_{\phi}$ as a function of the $\pi^{+} \pi^{-}$ invariant mass. As seen in Fig. 5 , the function $\mathcal{A}_{\phi}\left(t, s_{\pi}\right)$ has a similarity to the Stokes vector $S_{1}(t, \omega)$ plotted in Fig. 1, confirming the expectation that the $C P$-odd, $T$-odd term in the $\phi$-distribution of $K^{0} \rightarrow \pi^{+} \pi^{-} e^{+} e^{-}$is correlated with the $C P$-odd, $T$-odd component of the Stokes vector in $K^{0} \rightarrow \pi^{+} \pi^{-} \gamma$. 
Finally, the spectrum-integrated decay rate in $K^{0} \rightarrow \pi^{+} \pi^{-} e^{+} e^{-}$and $\overline{K^{0}} \rightarrow$ $\pi^{+} \pi^{-} e^{+} e^{-}$contains the standard $K_{L}-K_{S}$ interference effect proportional to $\eta_{+-}$, and a corresponding asymmetry between $K^{0}$ and $\overline{K^{0}}$, which is shown in Fig. 6.

\section{Additional Remarks}

(i) Our analysis of the time-dependence in $K^{0}, \overline{K^{0}} \rightarrow \pi^{+} \pi^{-} e^{+} e^{-}$assumed the amplitude to be entirely determined by the radiative decay $K^{0}, \overline{K^{0}} \rightarrow$ $\pi^{+} \pi^{-} \gamma$. A non-radiative contribution to the amplitude $K_{L} \rightarrow \pi^{+} \pi^{-} e^{+} e^{-}$is possible in the form of a "charge-radius" term, with the characteristic feature of producing $\pi^{+} \pi^{-}$in an $s$-wave. Such a configuration is not possible in the radiative decay $K_{L} \rightarrow \pi^{+} \pi^{-} \gamma$. We have investigated the effects of a charge radius term nominally parametrized by the coefficient $g_{P}=0.15 e^{i \delta_{1}}$ defined in [3]. It has the interesting consequence of inducing a small term of the form $K_{4} \sin 2 \theta_{l} \cos \phi$ in the angular distribution $d \Gamma / d \cos \theta_{l} d \phi$ given in Eq. (19). Such a term is $C P$-odd but $T$-even [3,7]. In Fig. 7, we show the magnitude and time-dependence of the coefficient $K_{4} / K_{1}$ generated by a charge-radius term $g_{P}$ with the nominal value given above.

(ii) Since the time-dependent interference effects in the decay spectrum of $K^{0}, \overline{K^{0}} \rightarrow \pi^{+} \pi^{-} e^{+} e^{-}$are strongest in the region $t \sim 10 \tau_{s}$, one can ask whether similar effects could be observed with a beam of the form $K_{L}+\rho K_{S}$, where $\rho$ is a regeneration amplitude induced by passage of $K_{L}$ through material. We have calculated the decay spectrum of $\left(K_{L}+\rho K_{S}\right) \rightarrow \pi^{+} \pi^{-} e^{+} e^{-}$as a function of time, for some typical regeneration parameters $\rho=|\rho| e^{i \varphi_{\rho}}$, such as $|\rho|=0.002,0.02$ and 0.2 with $\varphi_{\rho}=-\pi / 4$. In particular, the time-dependent asymmetry $\mathcal{A}_{\phi}$ for such a beam is shown in Fig. 8. It is clear that a suitable choice of a regenerator would permit a study of the $C P$-odd, $T$-odd feature in the $\pi^{+} \pi^{-} e^{+} e^{-}$decay of a neutral $\mathrm{K}$ meson, as well as other aspects of the decay spectrum.

(iii) Several refinements in the matrix element of $K^{0}, \overline{K^{0}} \rightarrow \pi^{+} \pi^{-} e^{+} e^{-}$can be imagined. There is a small short-distance contribution associated with the interaction $s \bar{d} \rightarrow e^{+} e^{-}$[3], which, however, is highly suppressed on account of the small quark mixing factor $V_{t s} V_{t d}^{*}$. The magnetic dipole term in the radiative amplitude $K_{L} \rightarrow \pi^{+} \pi^{-} \gamma$, represented by the coupling constant $g_{M 1}$, actually has a certain dependence on $s_{\pi}$ [8], which can be incorporated into the analysis. Finally, the radiative amplitudes $K_{L, S} \rightarrow \pi^{+} \pi^{-} \gamma$ could contain additional components such as a direct $E 1$ multipole in $K_{S}$ decay or a direct $E 2$ term in $K_{L}$ decay (see, e.g. [9]). The former would give rise to a departure from pure bremsstrahlung in the photon energy spectrum of $K_{S} \rightarrow \pi^{+} \pi^{-} \gamma$, while the latter would produce a $C P$-violating charge asymmetry in the Dalitz 
plot of $K_{L} \rightarrow \pi^{+} \pi^{-} \gamma$. There is also the possibility of a direct $C P$-violating $E 1$ multipole in $K_{L}$ decay, that would show up as a difference between $\eta_{+-\gamma}$ and $\eta_{+-}$in the time-dependence of $K_{L}+\rho K_{S} \rightarrow \pi^{+} \pi^{-} \gamma$.

A specific consequence of introducing direct $E 1$ multipoles in $K_{L} \rightarrow \pi^{+} \pi^{-} \gamma$ is to modify the amplitude $E_{L}$ in Eq.(5) to

$$
E_{L}=\left(\frac{2 M_{K}}{\omega}\right)^{2} \frac{\eta_{+-} e^{i \delta_{0}}}{1-\beta^{2} \cos ^{2} \theta}+g_{E 1} e^{i\left(\varphi_{+-}+\delta_{1}\right)}+i \tilde{g}_{E 1} e^{i \delta_{1}}
$$

where $g_{E 1}$ is a measure of direct (CP-conserving) $E 1$ emission in $K_{S} \rightarrow$ $\pi^{+} \pi^{-} \gamma$, and $\tilde{g}_{E 1}$ a measure of a direct $C P$-violating $E 1$ emission in $K_{L} \rightarrow$ $\pi^{+} \pi^{-} \gamma\left(g_{E 1}\right.$ and $\tilde{g}_{E 1}$ being real). The resulting change in the asymmetry $\mathcal{A}_{\phi}$ in $K_{L} \rightarrow \pi^{+} \pi^{-} e^{+} e^{-}$is $[2]$

$$
\mathcal{A}_{\phi}=15 \% \sin \left(\varphi_{+-}+\delta_{0}-\delta_{1}\right)+38 \%\left[\frac{g_{E 1}}{\left|g_{M 1}\right|} \sin \varphi_{+-}+\frac{\tilde{g}_{E 1}}{\left|g_{M 1}\right|}\right] \text {. }
$$

The observed branching ratio for $K_{S} \rightarrow \pi^{+} \pi^{-} \gamma$ limits $\left|g_{E 1} / g_{M 1}\right|$ to $<5 \%$ [2], while typical estimates of $\left|\tilde{g}_{E 1} / g_{M 1}\right|$ are of order $10^{-3}[9]$.

Needless to say, any incisive study of the channels $K^{0}, \overline{K^{0}} \rightarrow \pi^{+} \pi^{-} e^{+} e^{-}$and $K_{L, S} \rightarrow \pi^{+} \pi^{-} \gamma$ should be alert to the possible presence of correction terms of the form described above.

\section{References}

[1] KTeV Collaboration, A. Alavi-Harati et al., Phys. Rev. Lett. 84, 408 (2000).

[2] L. M. Sehgal and M. Wanninger, Phys. Rev. D 46, 1035 (1992); 46, 5209(E) (1992).

[3] P. Heiliger and L. M. Sehgal, Phys. Rev. D 48, 4146 (1993); 60, 079902(E) (1999).

[4] E. J. Ramberg et al., Fermilab Report No. FERMILAB-CONF-91-258, 1991; E731 Collaboration, Phys. Rev. Lett. 70, 2525 (1993).

[5] L. M. Sehgal and J. van Leusen, Phys. Rev. Lett. 83, 4933 (1999).

[6] M. Contalbrigo, NA48 Collaboration, talk given at XXXVth Rencontres de Moriond (Les Arcs, March 11-18, 2000).

[7] L. M. Sehgal, to appear in Kaon Physics, edited by J. L. Rosner and B. D. Winstein (The University of Chicago Press, Chicago and London); hepph/9908338. 

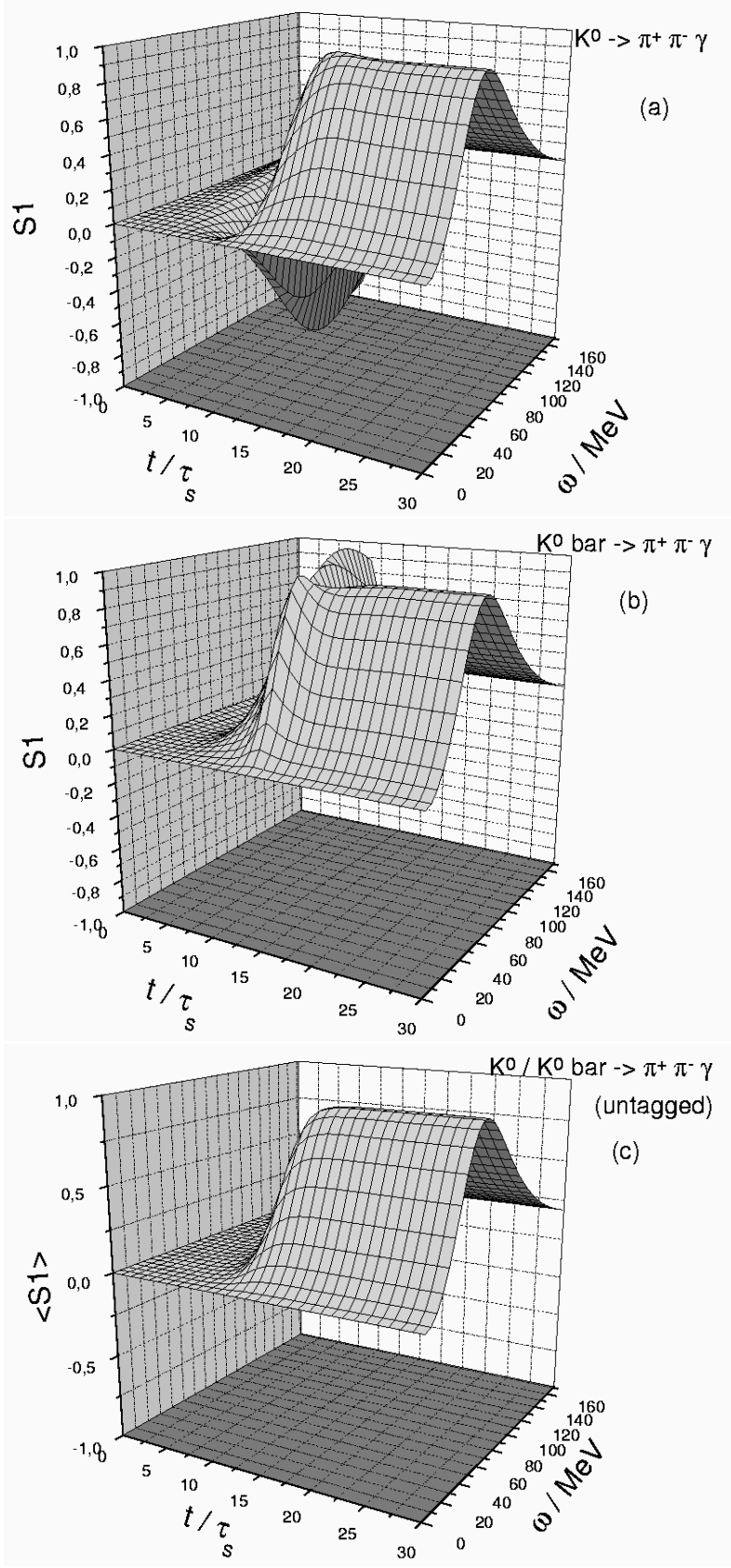

Fig. 1. (a) Component $S 1$ of the Stokes vector of the photon as a function of photon energy and time for the decay $K^{0} \rightarrow \pi^{+} \pi^{-} \gamma$; (b) same as (a) but for initial $\overline{K^{0}}$; (c) same as (a) but for an incoherent equal mixture of $K^{0}$ and $\overline{K^{0}}$.

[8] B. Cox, KTeV Collaboration, in Proceedings of the International Workshop on $C P$ Violation in K, edited by S. Sugimoto and T. Yamanaka (KEK, Tokyo, 1999), pp. 59-70; J. Belz, hep-ex/9903025.

[9] G. D'Ambrosio and G. Isidori, Int. J. Mod. Phys. A 13, 1 (1998) and references therein. 

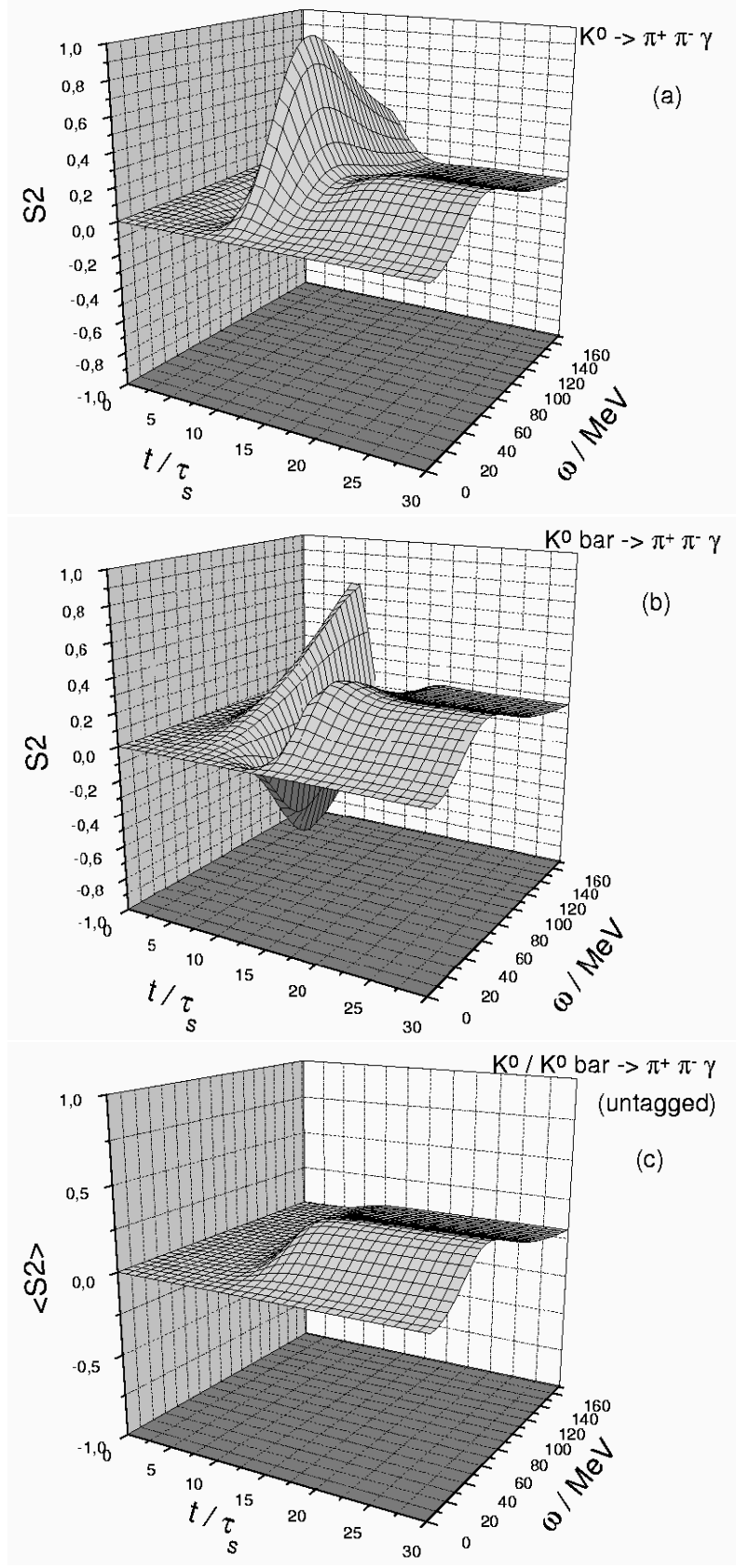

Fig. 2. (a) Component $S 2$ of the Stokes vector of the photon as a function of photon energy and time for the decay $K^{0} \rightarrow \pi^{+} \pi^{-} \gamma$; (b) same as (a) but for initial $\overline{K^{0}}$; (c) same as (a) but for an incoherent equal mixture of $K^{0}$ and $\overline{K^{0}}$. 


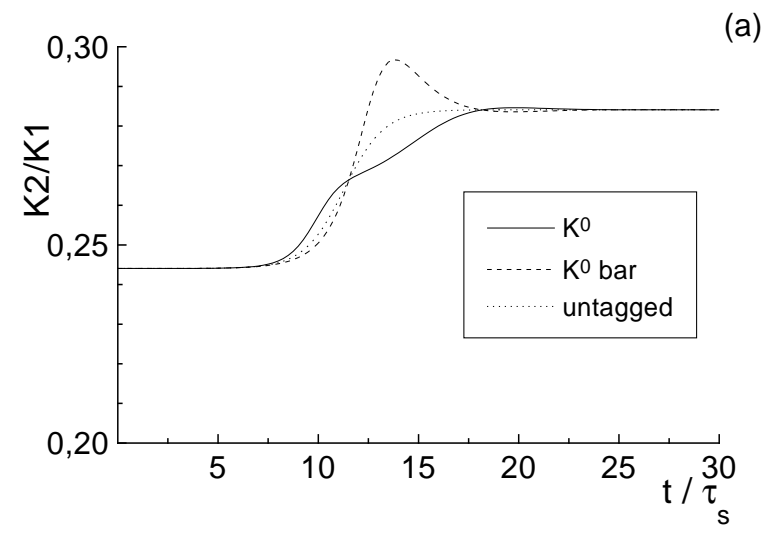

(a)

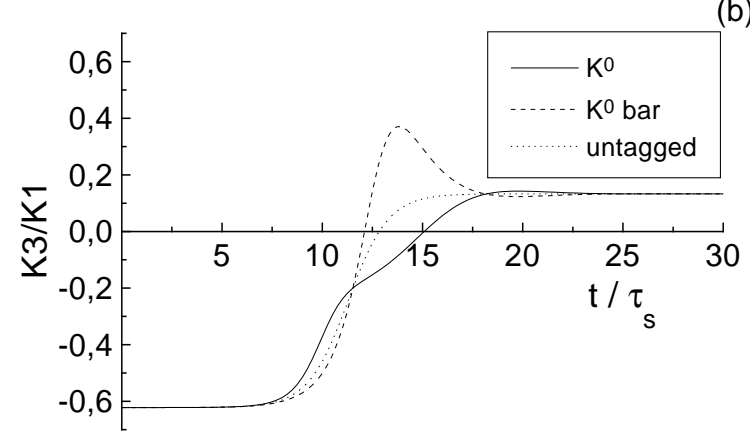

(b)

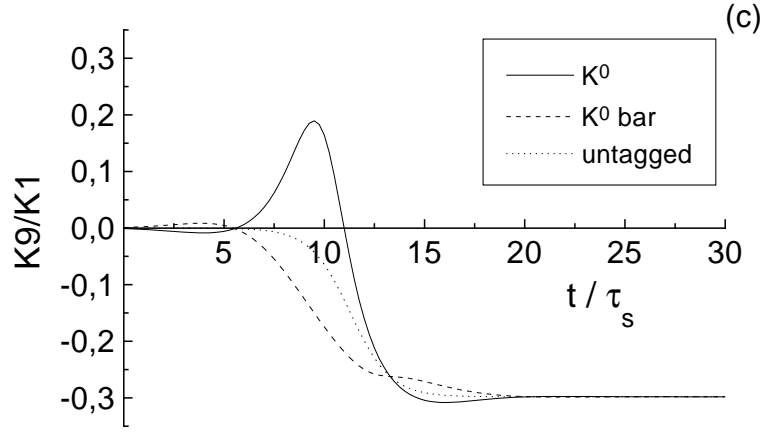

Fig. 3. Time-dependent coefficients $K_{i} / K 1$ : (a) $K 2 / K 1$ for the decays $K^{0}$ and $\overline{K^{0}} \rightarrow \pi^{+} \pi^{-} e^{+} e^{-}$as well as for an untagged initial beam; (b) same as (a) but for $K 3 / K 1$; (c) same as (a) but for $K 9 / K 1$. 


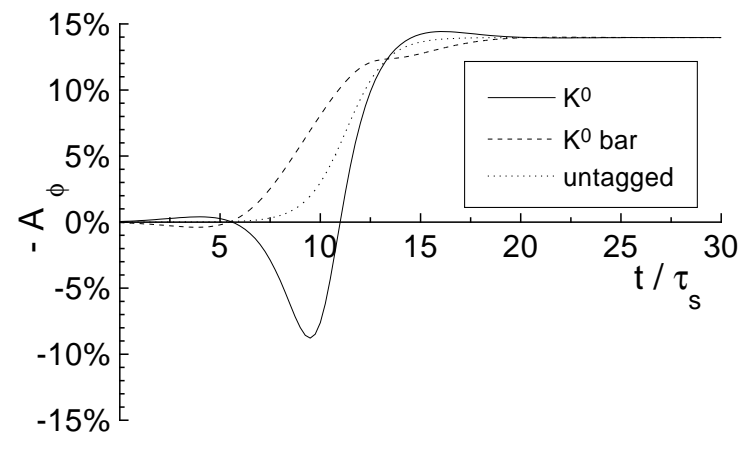

Fig. 4. Time-dependent Asymmetry $\mathcal{A}_{\phi}$ for the decays $K^{0}$ and $\overline{K^{0}} \rightarrow \pi^{+} \pi^{-} e^{+} e^{-}$as well as for an incoherent equal mixture. 

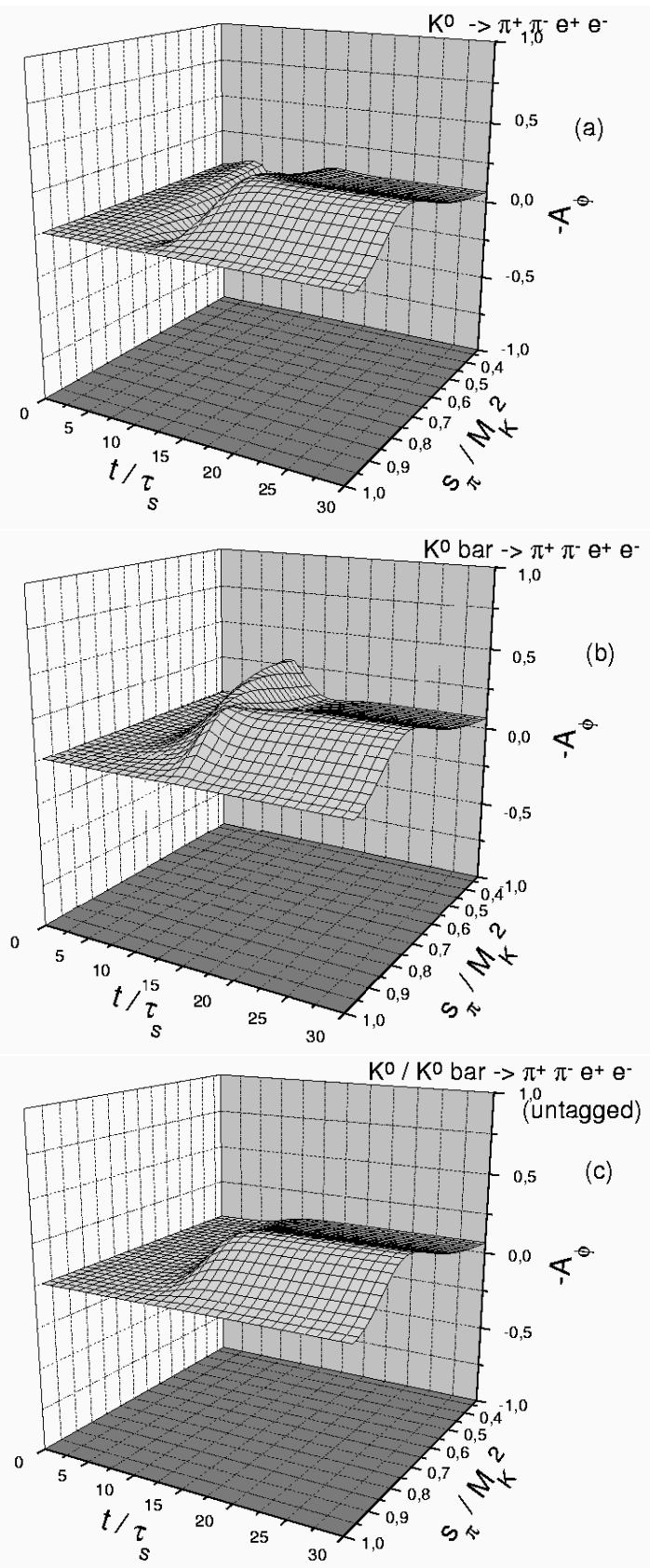

Fig. 5. (a) The asymmetry $\mathcal{A}_{\phi}$ as a function of $s_{\pi}$ and time for the decay $K^{0} \rightarrow \pi^{+} \pi^{-} e^{+} e^{-}$; (b) same as (a) but for initial $\overline{K^{0}}$; (c) same as (a) but for an incoherent equal mixture of $K^{0}$ and $\overline{K^{0}}$. 

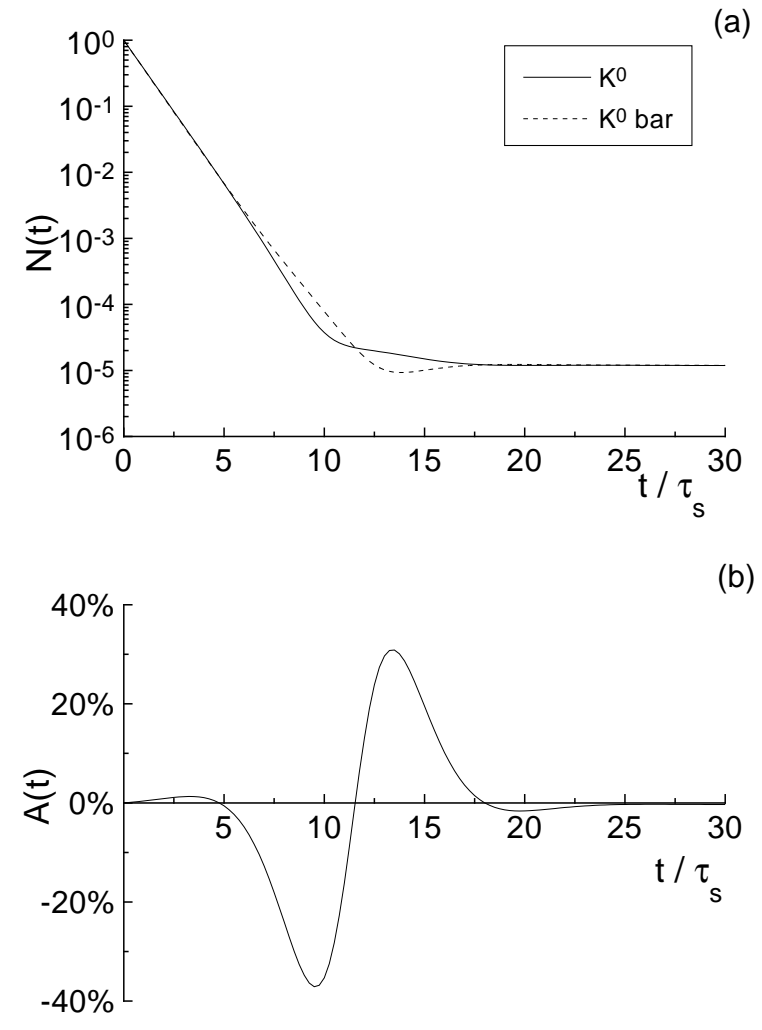

Fig. 6. (a) Spectrum integrated decay rate for $K^{0}$ and $\overline{K^{0}} \rightarrow \pi^{+} \pi^{-} e^{+} e^{-}$; (b) asymmetry in the decay rate $\mathcal{A}(t)=(N(t)-\bar{N}(t)) /(N(t)+\bar{N}(t))$.

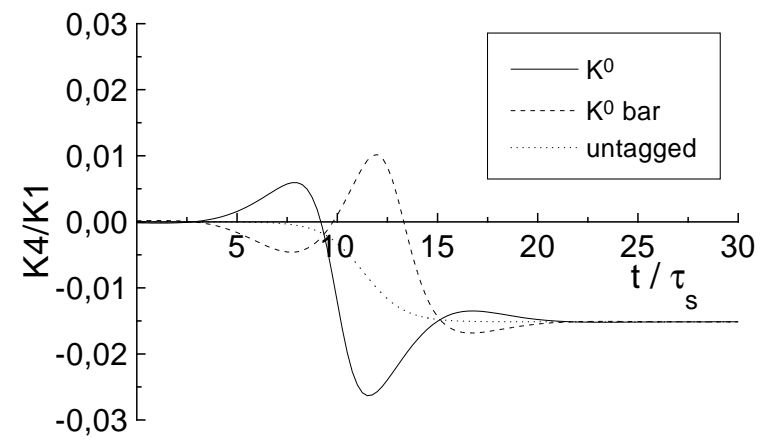

Fig. 7. Coefficient $K 4 / K 1$ generated by a charge-radius term for $K^{0} \rightarrow \pi^{+} \pi^{-} e^{+} e^{-}$, $\overline{K^{0}} \rightarrow \pi^{+} \pi^{-} e^{+} e^{-}$and an untagged initial beam. 


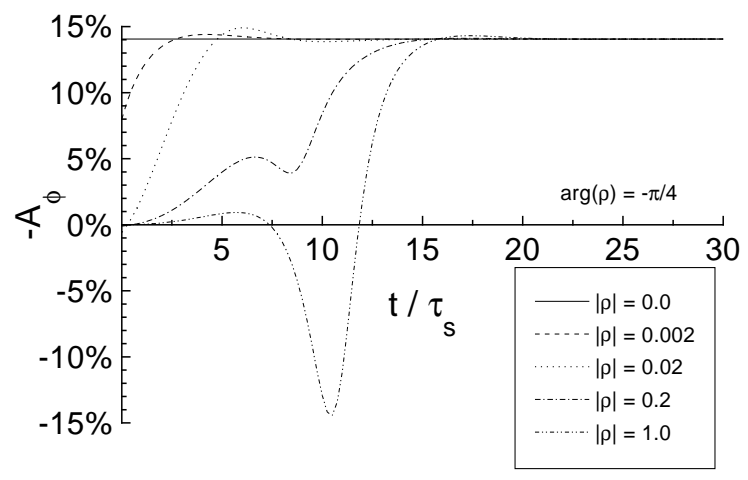

Fig. 8. Time-dependent asymmetry $\mathcal{A}_{\phi}$ for a regenerated beam $K_{L}+\rho K_{S}$. 